International Journal of Biomedicine | June 2021 - Volume 11, Issue Suppl_1: Abstracts from the Third Russian International Conference "Cryo-electron microscopy 2021: achievements and prospects"

POSTER ABSTRACT PRESENTATIONS

SESSION TITLE: EM RESEARCH RELATED TO MEDICINE

DOI: 10.21103/IJBM.11.Suppl_1.P35

\title{
Abstract P-35: Cryo-Electron Tomography of Protein Conjugated Upconverting Nanophosphors
}

\author{
Vyacheslav A. Kralin $^{1}$, Anton S. Orekhov ${ }^{1,2}$, Roman A. Kamyshinsky ${ }^{1,2}$, \\ Yury M. Chesnokov ${ }^{2}$, Polina A. Demina ${ }^{3}$ \\ ${ }^{1}$ Institute of Physics and Tecknology, Moscow, Russia \\ ${ }^{2}$ National Research Centre "Kurchatov Institute," Moscow, Russia \\ ${ }^{3}$ Shemyakin-Ovchinnikov Institute of Bioorganic Chemistry, RAS, \\ Moscow, Russia
}

Background: Over the past decades, significant advances have been made in the field of creating nanobioreagents for solving modern medicine problems (Grebenik et al., JBO, 2013). However, the problem of their low accumulation rate in pathological tissue in vivo experiments still remains. First of all, it is associated with the adsorption of blood proteins on the surface of nanobioreagents and the protein layer formation, which significantly changes the surface properties, which leads to their rapid excretion by the reticuloendothelial system. In particular, it is possible to reduce the blood plasma proteins adsorption and increase the time spent in the circulatory system by forming a coating of proteins.

Methods: In situ cryoelectron tomography (Cryo-ET) is the only method that allows the experimental observation of protein structures on the nanoparticle's surface in their natural functional state. The basic principle of the method is to obtain a series of projections of a vitrified sample thin lamella at different tilt angles related to an incident electron beam. Their further processing leads to obtaining the volumetric information about the structure of the sample. The use of a cryo-focused ion beam (Cryo-FIB) in specimen thinning makes it possible to carry out experiments with thin sections of cellular structures and observe the penetration of nanoparticles into the intracellular environment.

Results: Upconverting nanophosphors (AN) were used as a nanoplatform for creating a protein coating. To create a protein coating on the AN surface, they were functionalized using an amphiphilic polymer containing carboxyl groups. 
Then, conjugation with protein molecules from the class of immunoglobulins was carried out by the method of carbodiimide activation.

At each stage of synthesis and modification, AN solutions with different size distribution were vitrified for subsequent tomography. After a series of experiments to study the morphology of nanoparticles, an experiment on their successful absorption by cells of the cancer line A549 was carried out.

Conclusion: Within this work, a series of in situ Cryo-ET methods were proposed and applied for structural characterization and visualization of the processes of synthesis, modification, and engulfment of nanoparticles into cellular systems. For the first time in its native form, the engulfment of ANF into the internal environment of the A549 cancer line cells was demonstrated.

Key Words: cryo-tomography $\bullet$ upconverting nanophosphors

This work was financially supported by the National Research Center "Kurchatov Institute" (order dated 02.07.2020 No. 1056)

*Corresponding author: Vyacheslav Kralin. E-mail: kralin.va@phystech.edu

International Journal of Biomedicine. 2021;11 Suppl 1: S27.

doi: 10.21103/IJBM.11.Suppl_1.P35

(C)2021 International Medical Research and Development Corporation 